Éducation

et sociétés

plurilingues

\section{Éducation et sociétés plurilingues}

36 | 2014

Varia

\title{
Autour d'un cas de bilinguisme scolaire italo-français, par Hélène David
}

Mathilde Anquetil

\section{(2) OpenEdition}

\section{Journals}

Édition électronique

URL : http://journals.openedition.org/esp/169

DOI : 10.4000/esp.169

ISSN : 2532-0319

Éditeur

Centre d'Information sur l'Éducation Bilingue et Plurilingue

\section{Édition imprimée}

Date de publication : 1 juin 2014

Pagination : 104-106

ISSN : 1127-266X

\section{Référence électronique}

Mathilde Anquetil, «Autour d'un cas de bilinguisme scolaire italo-français, par Hélène David », Éducation et sociétés plurilingues [En ligne], 36 | 2014, mis en ligne le 26 octobre 2014, consulté le 24 septembre 2020. URL : http://journals.openedition.org/esp/169 ; DOI : https://doi.org/10.4000/esp.169 


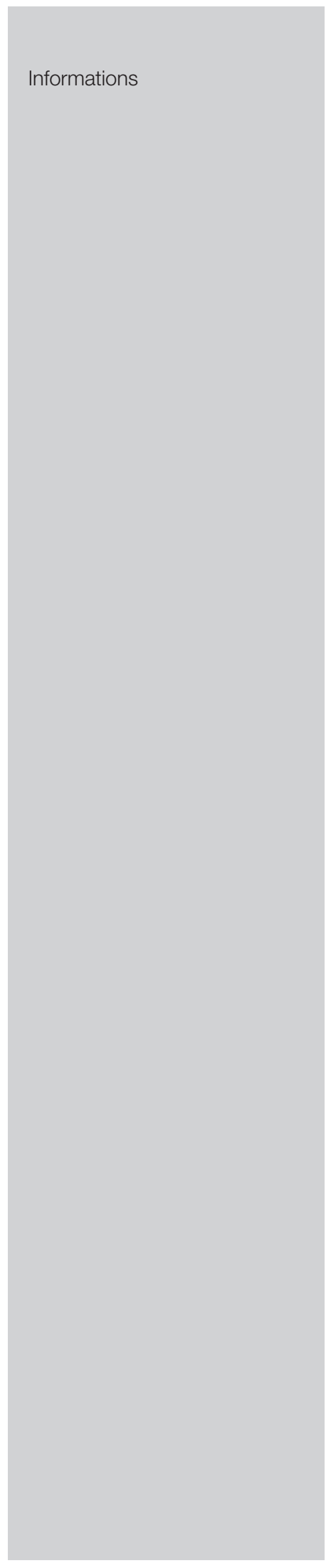

\section{Lecture de thèse}

Hélène DAVID. 2013. Autour d'un cas de bilinguisme scolaire italo-français: discours, contextes et idéologies au miroir des voix d'adolescents-apprenants. Un parcours de chercheure pour une réflexion approfondie en matière de politiques linguistiques et d'éducation au plurilinguisme. Thèse de doctorat (sous la dir. de Danielle LÉVY). Scuola dottorale dell'Università di Macerata. Giclo XXIV. Corso di Dottorato di Ricerca in Politica, Educazione, Formazione Linguistico-Culturali. Mathilde ANQUETIL

La thèse soutenue par Hélène David, enseignante de la section internationale à option française du Lycée Galvani de Bologne (Italie), intéressera le lecteur de la revue Education et Sociétés plurilingues à plus d'un titre. Située dans le contexte de l'éducation bilingue italo-française, elle renouvelle les études en concentrant son attention sur des acteurs peu entendus dans les dispositifs montés à leur bénéfice: les apprenants eux-mêmes. Comment se situent-ils dans le flot discursif institutionnel qui accompagne la volonté de promouvoir les cursus internationaux? Quelle voix émerge des adolescents-apprenants face aux politiques éducatives dont ils sont l'objet et qu'ils réinvestissent de leur représentations et de leur vécu? Et en miroir, quel parcours réflexif est amené à construire l'enseignant qui se met en posture de chercheur sur son propre terrain?

Le travail d'H. David se base sur une étude diachronique de la coopération linguistique et éducative franco-italienne et des facteurs de son évolution (représentations des langues, stratégies de leur diffusion; dynamique de la construction européenne et ses enjeux politiques et idéologiques), aboutissant sur le terrain à des dispositifs bilingues qui, avec la récente institution de l'ESABAC, passent des quelques sections d'élite à une diffusion plus démocratisée mais dont les nœuds problématiques didactiques et conceptuels, ne sont pas tous déliés. De ce point de vue, le lycée Galvani constitue un laboratoire et un observatoire particulier avec ses quelque vingt années d'expérience pilote.

Mais une fois explicité ce contexte, l'objectif de la jeune enseignante-chercheure est de saisir les logiques d'actions individuelles en se mettant à l'écoute des sujets sans se soustraire à la tension entre implication et mise à distance interprétative des propos des jeunes en formation. Le corpus recueilli de 16 entretiens semi- 
directifs approfondis fait l'objet d'une analyse thématique: le français et les langues en contact, l'expérience en section bilingue italo-française, l'identité plurilingue, la valorisation individuelle et sociale, l'Europe en expérience et représentations. Mais en croisant ces thématiques émergentes avec l'analyse du discours, l'analyse des représentations et des questionnements identitaires et l'analyse de la communication interculturelle, la grille d'interprétation se raffine sur des problématiques qui interpellent les acteurs du terrain de l'éducation bilingue pour penser la programmation:

- Comment l'adolescent-apprenant perçoit-il et intègre-t-il les pratiques dominantes en langues dans un monde globalisé?

- Quelles interprétations les acteurs livrent-ils de leur pratiques plurilingues, Quelle conscientisation métalinguistique atteignentils?

- Comment les apprenants de la classe bilingue interprètent-ils et gèrent-ils leur différence? Quelle lecture interculturelle produisent-ils de cette expérience?

- Excellence et performance sont-elles moteur de l'éducation bilingue?

Sur chacune de ces questions H. David instaure un dialogue entre les paroles des apprenants et ses propres catégorisations interprétatives pour interroger le dispositif et ses pratiques, afin de formuler des implications quant aux politiques d'aménagement éducatif, ainsi qu'une série de propositions pragmatiques.

L'éducation bilingue italo-française gagnerait ainsi à être thématisée de façon plus approfondie sur les thèmes des représentations croisées et sur la communication interculturelle franco-italienne contemporaine, mais aussi sur la réflexion métalinguistique en approche comparée avec une dimension socio-linguistique; elle gagnerait aussi à se dégager du couple binaire pour être pensée dans son insertion dans un monde vu comme espace d'action plurilingue globalisé qui comprend explicitement l'anglais.

L'élargissement du public ESABAC nécessite par ailleurs que l'on diversifie les programmes selon les filières et que l'on inscrive davantage l'agir social, la pédagogie du projet et de l'échange non seulement pour mieux converger avec la perspective actionnelle promue par le CECR, mais pour répondre aux motivations et perspectives des jeunes qui s'engagent dans un parcours exigent.

Espérons que le travail d'H. David fera prochainement l'objet d'une publication pour la diffusion de ces résultats, fruits d'une analyse qui allie solidité des bases théoriques, souplesse de l'écou- 


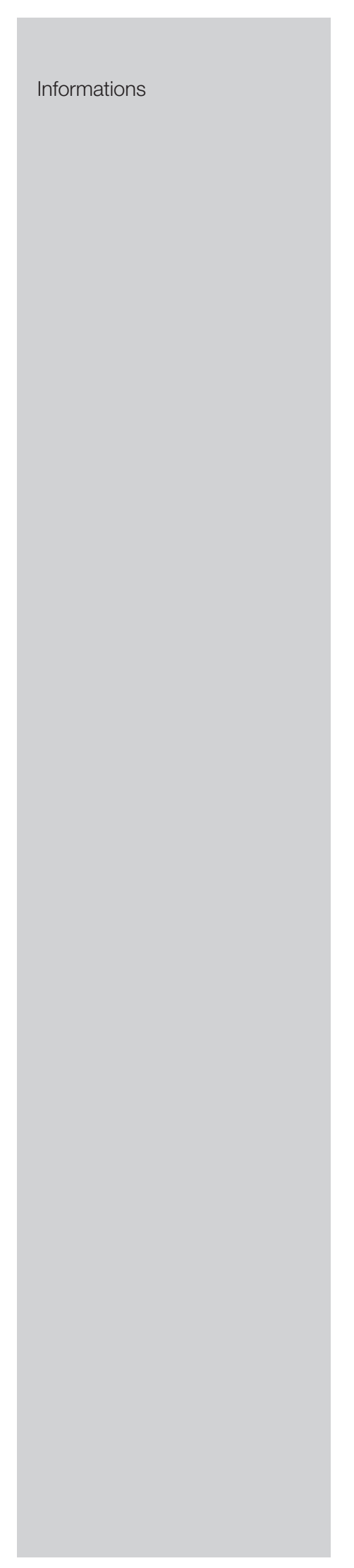

te des subjectivités en jeu dans les voix des adolescents, veille critique vis-à-vis des discours idéologiques mais aussi souci d'apporter des éléments pragmatiques en retour de la recherche sur le terrain.

Pour plus d’informations: helene.david@unimc.it 